\title{
THE IMPACT OF PACS (PICTURE ARCHIVING AND COMMUNICATION SYSTEM) ON HOSPITAL WORKFLOW: A SYSTEMATIC REVIEW
}

\author{
Reza Khajouei ${ }^{1}$, Misagh Zahiri Esfahani ${ }^{2 *}$
}

1: PhD in Medical Informatics, Medical Informatics Research Center, Institute for Futures Studies in Health, Kerman University of Medical Sciences, Kerman, Iran.

2: MSc Student of Health Information Technology, Student Research Committee, Kerman University of Medical Sciences, Kerman, Iran.

Correspondence:

Tel: +98.9901876836, Fax: +98. 34-31325415, E-mail: misaghzahiri@yahoo.com

\section{TYPE OF ARTICLE: CONFERENCE ABSTRACT}

\begin{abstract}
Introduction: Today, PACS is used as a digital system for storing, disseminating, management, and presentation of medical images in health care settings. An emerging new digital system can influence users' workflow. However, the effect of PACS on work practice changes in radiology departments is not entirely clear. The objective of this study is to systematically review the literature to understand the effect of PACS on the workflow of its users.

Methods: We systematically searched all English papers on evaluation studies of PACS published in the last 25 years, starting in 1990. We searched PubMed and Science Direct databases for relevant papers with three groups of key terms. The first group consisted of terms related to evaluation, the second group related to PACS, and the third group related to workflow. To capture studies about the evaluation of PACS, the key terms were used in the following two-step process: 1) key terms in each group were combined by the operator "OR"; 2) groups A, B, and $\mathrm{C}$ were combined using the operator "AND". Two reviewers independently reviewed and assessed titles and abstracts of the resulting papers against predefined inclusion and exclusion criteria. Full-text articles were retrieved and examined to identify final eligible studies.

Results: In this study, 10 out of 121 retrieved articles (PubMed $=74$ and Science Direct $=47)$ met our inclusion criteria and were reviewed by the authors. Eight studies reported positive effects of PACS on workflow. The way that articles addressed the workflow in seven studies measured aspects of time associated with the introduction of PACS: namely, the availability of images, the time a physician took to review an image, reduction in reporting time, time to access images, and searching time of images. Three other studies addressed the workflow by measuring satisfaction, cost, and human savings.

Conclusion: The results of this study showed that PACS can improve hospital workflow by reducing the time of reviewing, accessing, searching, and reporting medical images and improving the efficiency of work practice. Based on the results, it is suggested that further studies are necessary to address efficacy of PACS as well as impact of PACS on users' satisfaction, cost, and human savings.

KEYWORDS: PACS, picture archiving and communication systems, Workflow, Work practice, Efficiency
\end{abstract}

\section{Abstracts of First National Congress of Medical Informatics, Mashhad, Iran, February 2017}

(C) 2017 The Authors. This is an open access article under the terms of the Creative Commons Attribution-NonCommercialNoDerivs License, which permits use and distribution in any medium, provided the original work is properly cited, the use is non-commercial and no modifications or adaptations are made. 